\title{
Genetic advances will influence the practice of medicine: Examples from cancer research and care of cancer patients
}

Gilbert S. Omenn, $M D, P h D$

\begin{abstract}
Exciting new techniques of genomics, proteomics, and bioinformatics are beginning to influence the practice of medicine, most notably in diagnosis and drug development for patients with various cancers. Examples are drawn from B-cell lymphomas, melanomas, and prostate, lung, and breast cancers. As in all evidence-based clinical practice, physicians will be better prepared if they understand the nature of the tests and the kinds of information from which they and their consultants will make clinical inferences and assist patients in making clinical decisions. Physicians also can help put new technologies in cultural and ethical context. Genet Med 2002:4(6, Supplement):
\end{abstract} 15S-20S.

Key Words: genomics, proteomics, cancers

New techniques of gene expression microarrays, comparative genomics, proteomics, and bioinformatics are beginning to influence the practice of medicine, most notably in oncology. These developments are exciting, yet very complicated for physicians in practice. Thus it is important to note that the physicians will not be conducting these new tests themselves, but will be relying on clinical pathology laboratories and special genetic testing laboratories. Nevertheless, as in all evidence-based clinical practice, it is helpful to understand the nature of the tests and the kinds of information from which we will make clinical inferences and assist patients in making clinical decisions.

\section{Aims of clinical applications of genomics and proteomics in oncology}

A crucial starting point is the recognition that we should always think and speak of cancers in the plural. The causes and pathogenesis of various cancers are heterogeneous (see Table 1). A major mistake in addressing physicians and other health professionals, and the public and the media, is to describe cancers as if there were one kind, one cause, or one type of treatment for all cancers. That message seemed to take root with "The War on Cancer" beginning in 1970, led by President Nixon and Senator Kennedy, with major increases in funding for the US National Cancer Institute research programs and high hopes of major progress against "cancer." One idea of the time was that viruses might be responsible for most cancers.

From the University of Michigan Health System, Ann Arbor, Michigan.

Gilbert S. Omenn, MD, PhD, University of Michigan Health System, 7324 Medical Sciences I Bldg., Ann Arbor, MI 48109-0626.

Received: July 11, 2002.

Accepted: September 23, 2002.

DOI: 10.1097/01.GIM.0000041436.07223.B0
Lack of understanding among scientists, physicians, media, and the public of the marked heterogeneity among cancers and multiple causes of cancers led to slow progress clinically, despite major progress in cancer biology and epidemiology, which frustrated many professionals and laypeople. Against some cancers, progress has been excellent; for many others, much less. Unfortunately, the greatest progress has been against the less common cancers.

A corollary of heterogeneity is to recognize predispositions to specific types of cancers. Molecular techniques are revealing details of precursor lesions and biomarkers that can become molecular signatures. The aims are to diagnose earlier and to treat more specifically, with a greater therapeutic margin of benefit to adverse effects. We should be able to intervene much more selectively and to protect more patients from adverse effects.

The heterogeneous molecular signaling pathways-revealed with studies of mRNA and protein expression patterns, receptors, serum biomarkers, and agents to attack specific molecular targets-are useful for research on mechanisms, as well as for diagnosis, treatment, and prevention. Pharmacogenomic strategies for drug development aim at greater selectivity for identifiable subpopulations.

Epidemiological data about incidence, prevalence, and mortality of cancers are available from the World Health Organization's International Agency for Research on Cancer. ${ }^{1}$ It is useful to consider the cancers with the greatest public health impact on a population basis, especially when engaging colleagues from other countries. An example is shown in Table 2, comparing ranking of cancer causes of death for Korea and the United States, as was appropriate for the international symposium at which this paper was originally presented (third biennial Asan Medical Center/Harvard Medical International Symposium "Genomics and Proteomics: Impact on Medicine and Health," Seoul, Korea, July 3-4, 2001). For both men and 
Table 1

Applications of genomics in clinical oncology

- Delineate heterogeneity of etiology and pathogenesis of various cancers

- Recognize predispositions to cancers of many types

- Detect precursor lesions and molecular changes

- Treat more specifically with greater therapeutic margins

- Intervene selectively in new, more effective ways

- Protect patients from adverse effects of treatments

Table 2

Leading cancer causes of death in Korea and in the United States: Estimates for 2000

\begin{tabular}{llll}
\hline & \multicolumn{3}{c}{ Korea } \\
\hline Men & No. & \multicolumn{1}{c}{ Women } & No. \\
\hline Stomach & 8,675 & Stomach & 4,793 \\
Liver & 8,614 & Lung & 2,569 \\
Lung & 7,832 & Liver & 2,540 \\
Colon/rectum & 1,767 & Colon/rectum & 1,518 \\
Esophagus & 1,492 & Cervix & 1,469 \\
Pancreas & 1,421 & Breast & 1,024 \\
& & Pancreas & 955
\end{tabular}

\begin{tabular}{|c|c|c|c|}
\hline Total & 571 & Total & 19,967 \\
\hline \multicolumn{4}{|c|}{ United States } \\
\hline Men & No. & Women & No. \\
\hline Lung & 98,738 & Lung & 63,378 \\
\hline Prostate & 35,882 & Breast & 45,553 \\
\hline Colon/rectum & 29,843 & Colon/rectum & 29,753 \\
\hline \multirow[t]{2}{*}{ Pancreas } & 14,026 & Pancreas & 14,796 \\
\hline & & Ovary & 14,033 \\
\hline Non-Hodgkin lymphoma & 12,835 & Non-Hodgkin lymphoma & 11,406 \\
\hline Leukemias & 11,915 & Leukemias & 9,511 \\
\hline Total & 301,050 & Total & 268,965 \\
\hline
\end{tabular}

From IARC. ${ }^{1}$

women in Korea, the leading cancers are stomach, liver, and lung. In the United States, stomach and liver cancers are very much less common, while lung cancers are by far the leading cause of cancer deaths in both women and men, followed by breast or prostate, then colorectal and pancreatic cancers. Prostate does not appear among the common cancer causes of death in Korea.

For many of these common cancers new ways of characterizing clinical, cytogenetic, and molecular heterogeneity are emerging. The National Cancer Institute has led the way with the Cancer Genome Anatomy Project, aiming to establish the repertoire of genes expressed, together with the amount of gene products produced for each, in normal cells and various types and stages of cancer cells. The result can be called "the cell's fingerprint." 2

\section{NEW METHODS LEAD TO NEW CONCEPTS}

The use of the polymerase chain reaction to greatly amplify detection of gene expression, the dense application of oligonucleotides to specially prepared silicon chips to create microarrays, the robotized sequencing of genes and detection of singlenucleotide polymorphisms, the combination of twodimensional electrophoresis and mass spectrometry to fractionate and identify proteins, and many other technical advances have revolutionized the kinds of questions that can be asked about tumors and about patients harboring tumors. For decades, laboratory researchers have sought individual molecular markers of various cancers, and epidemiologists have analyzed putative risk factors and biomarkers for significant associations with specific cancers.

This approach has had a limited yield, with many first reports unconfirmed in subsequent studies. An overriding explanation is surely the heterogeneity of many of the cancers and the modest attributable risk associated with individual risk factors or biomarkers, including genetic polymorphisms, studied one at a time. Now that hundreds or thousands of genes can be examined for increased or decreased expression as mRNA (upregulation or down-regulation) simultaneously with microarray chips, it is feasible to search for patterns of change during carcinogenesis or metastasis or response to therapy. In turn, the resulting avalanche of data requires new methods of analysis, highly dependent on computer methods of bioinformatics. ${ }^{3}$ The current analytical and visualization methods are highly descriptive, facilitating clustering of genes that are upor down-regulated together. More and more of these genes are being identified with biochemical functions through annotations of the human genome, permitting assessment of the biological plausibility of the associations. Recently, statistical methods suitable for large-scale databases have been introduced, bringing considerably more power to these analyses. ${ }^{4,5}$

As will be illustrated below with examples from particular cancers, the gene expression microarray methods are now complemented by multiple methods for patterns of protein expression both in tumors and in serum or plasma.

\section{A FUNCTIONAL MODEL FROM CANCER BIOLOGY}

Hanahan and Weinberg ${ }^{6}$ have introduced a model of cancers that gives new emphasis to the highly complex cellular content of tumors. Instead of the reductionist view of a mass of homogeneous neoplastic cells (and their tumor cell line derivative), Hanahan and Weinberg point to the roles of vascular endothelial cells, fibroblastic (stromal) cells, and immune cells, as well as the neoplastic cells (themselves notably heterogeneous as clones evolve). They organize the many biochemical signaling and metabolic pathways into six important functions, as shown in Figure 1: self-sufficiency in growth signals, 


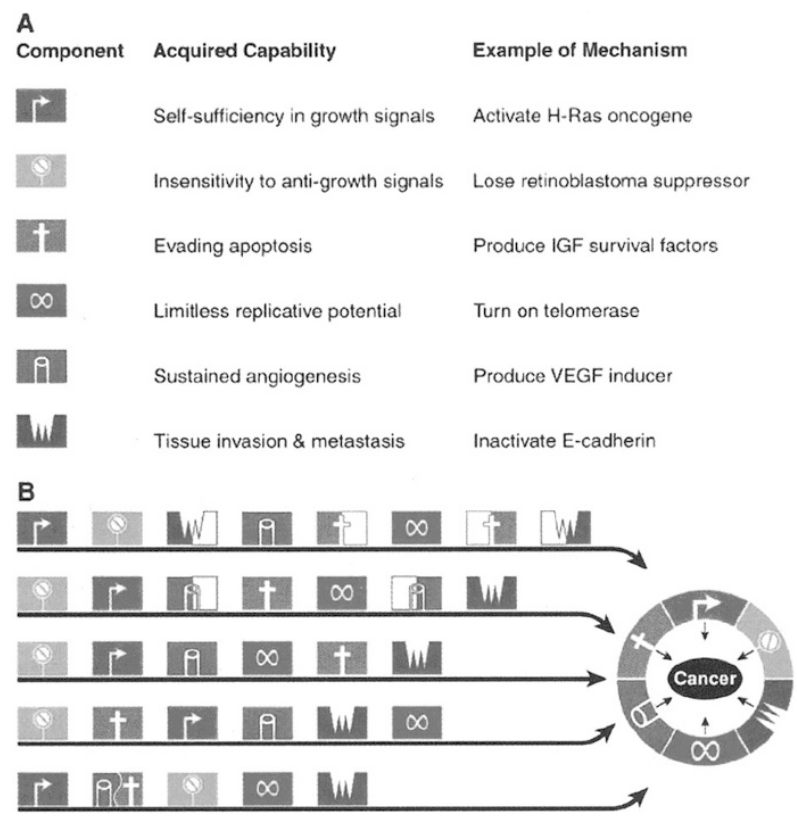

Fig. 1 Hanahan-Weinberg model of cancer biology, highlighting six phenomena with specific examples of mechanisms, in heterotypic tumors. Reprinted from Cell, ${ }^{6}$ copyright 2000, with permission from Elsevier Science.

insensitivity to antigrowth signals, evasion of apoptosis, unlimited replicative potential, sustained angiogenesis, and tissue invasion and metastasis. Great variation can arise from the nonlinear sequencing of expression of genes involved in each of these major phenomena.

It is timely to apply these organizing concepts to clusters of genes up-regulated and down-regulated in various stages of carcinogenesis and in response to preventive or therapeutic agents.

\section{SPECIFIC CANCERS}

\section{B-cell lymphomas}

A landmark publication by Alizadeh et al. ${ }^{7}$ from Stanford and six other institutions demonstrated the usefulness of gene expression gene chip methods in differentiating patients with different prognoses from chemotherapy against large B-cell lymphoma. First they characterized lymph node gene expression into certain patterns representing normal lymph nodes, the germinal centers of normal lymph nodes, proliferative zones of lymph nodes, and normal T cells. With some 10,000 oligonucleotides on the chips to hybridize and recognize expression of genes, a great many show differences from background or from the normal patterns. A convention was introduced to filter the results to show those genes whose expression is increased or decreased at least 2.5 times, and to suppress the mass of data on many genes whose expression varies little. In fact, this convention may introduce a serious problem. There are genes whose gene products are critical as rate-limiting steps for whole pathways, so that a change of much less than a factor of 2 or more might be quite important.
When diffuse large B-cell lymphoma gene expression patterns were then examined, two distinctly different patterns were identified, one resembling closely the germinal centers of $\mathrm{B}$ cells in lymph nodes and the other resembling the activated proliferation zone of B cells in lymph nodes. Of great clinical interest, these two patterns were associated with strikingly different survival curves: 9 deaths among 24 patients at "low" risk and 11 deaths among 14 patients at "high" risk $(P<0.002)$ over 8-10 years of follow-up. With additional pretreatment information, the survival rates could be stretched to $85 \%$ versus $15 \%$. Of course, for management of individual patients, it would be desirable to move as close to $100 \%$ versus $0 \%$ as feasible; nevertheless, these results can help identify a subgroup of patients not otherwise identifiable that can be expected to respond dramatically well to aggressive chemotherapy, while the other subgroup might be spared the toxicity of the treatment in light of very limited chance of benefit. It is hoped that a different treatment will now be devised and tested in clinical trials for the poorly responding subgroup. Without the molecular signatures, physicians and patients would remain committed to a therapy with efficacy in $40 \%$ of patients and toxicity in close to $100 \%$.

\section{Prostate cancers}

Rubin, Chinnaiyan, and colleagues at the University of Michigan have published extensive studies of molecular profiles of prostate cancer. ${ }^{8}$ Using methods similar to those of Alizadeh et al., they have classified carefully dissected prostate tissue specimens into normal adjacent tissue, benign prostatic hypertrophy, localized prostate cancer, and metastatic prostate cancer. Two control or comparison groups were used: first, a commercial pool of normal prostates from men with no cancers, and, second, normal adjacent prostate from cases with prostate cancers. As with any clinical test, it matters what the reference is for comparisons. Each type of specimen was differentiated by the microarray clustering method. Furthermore, many of the genes with marked up- or down-regulation fell into well-recognized categories of functions, including transcription factors, cell adhesion, protease/antiprotease, phosphatase/kinase, free radical scavenger, and inflammation/ immunity (Fig. 2).

In turn, the cancers have been classified as responsive or nonresponsive to antitestosterone (estrogenic) therapy. It is extremely useful clinically to differentiate hormone-refractory cases from hormone-responsive cases. In defining the molecular signatures for prostate cancers, they have combined tissue microarray and gene expression microarray. They also identified a specific biomarker (hepsin) that seems to have prognostic value for prostate cancers.

\section{Cutaneous malignant melanomas}

Bittner et al., ${ }^{9}$ in another multicenter study involving 11 different institutions, brought modern molecular techniques to this important cancer, whose incidence is rising steeply. Melanomas have no histopathological, molecular, or immunochemical markers to differentiate subsets of patients. There 


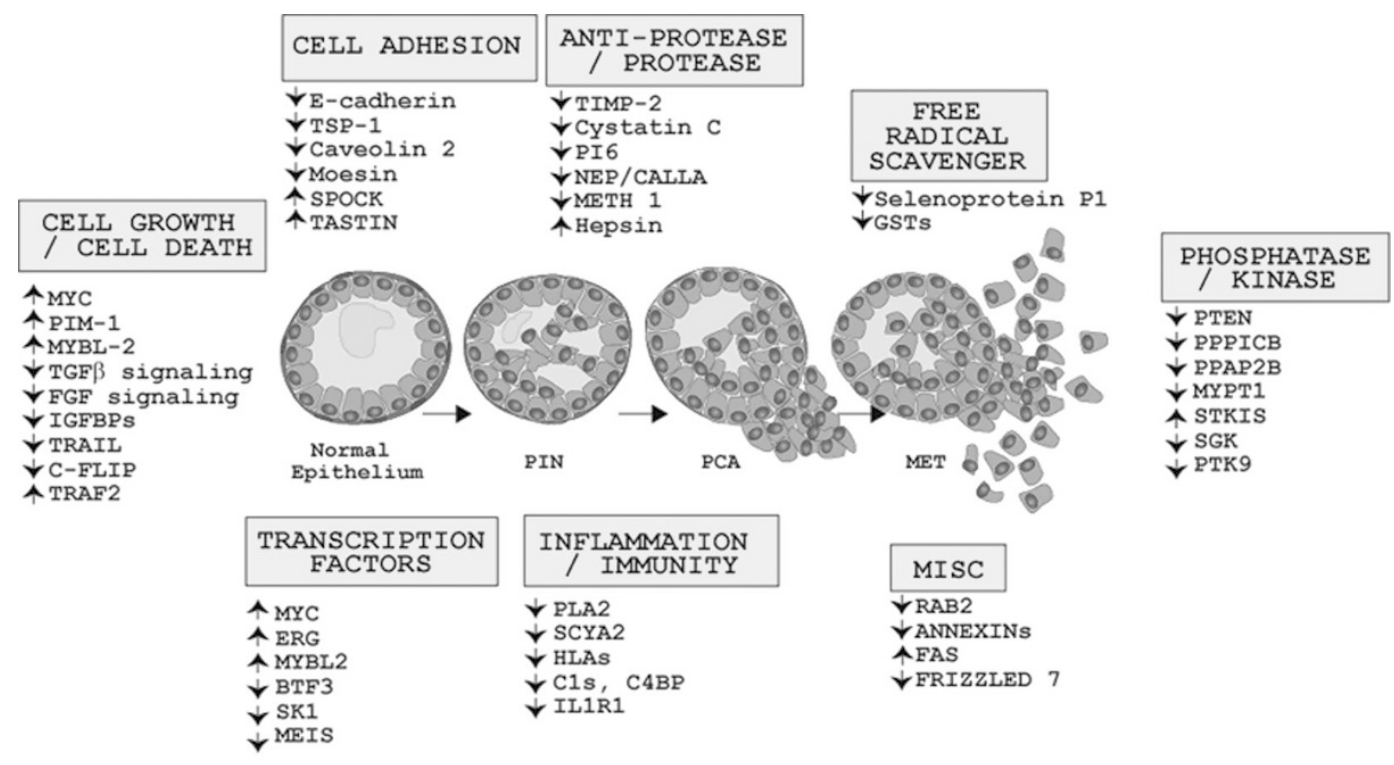

Fig. 2 Summary of significant changes in genes, by functional category, in the progression of molecular signatures from normal prostatic epithelium to prostate cancer in situ, to localized prostate CA, to metastatic prostate CA. Reproduced with permission from Nature, copyright $2001^{8}$ (http://www.nature.com).

are few known recurring genetic (cytogenetic) changes in these tumors. And nonsurgical treatment is notoriously ineffective in cases of advanced disease. A clever visualization of the clustering of gene expression data were presented, based on standard dendrograms of 31 patients (Fig. 3A); 19 were concentrated in a major cluster, while the rest were distributed rather widely (Fig. 3B). Just 22 genes accounted for much of the separation of the major cluster from the other specimens of which 19 have known functions.

A substudy examined cell lines from very invasive malignant melanomas of the uvula, together with the cutaneous melanomas, combining the molecular assays with bioassays. First, a simulated scratch wound was made in plated cells, to determine whether the cells would grow out, how far they would migrate, and how rapidly they would move away from the initial site. The cells from the invasive lesions migrated dramatically from the initial position. Second, these cells actively stimulated coagulation of collagen in the gel. Finally, the most invasive melanoma cells pushed a trabecular framework aside. With the 31 cutaneous melanoma specimens, these bioassays showed remarkable differentiation for the major cluster of 19 melanomas, which had down-regulation of several genes related to spreading, migration, and formation of focal adhesions (integrins, syndecan 4, vinculin). The mixed group of 12 other melanomas had an opposite pattern, with higher activities associated with invasiveness, notably fibronectin, a promigratory molecule. Preliminary survival data (7/10 vs. 1/5 for major vs. nonmajor clusters) showed some differences, but require more follow-up.

\section{Lung cancers}

For lung cancers, we can also consider proteomics results, leading more directly to potentially useful biomarkers. Cell lines and microdissected tumors are useful in developing bi- omarkers, which must be evaluated clinically and epidemiologically. The proteome is a highly dynamic compartment, regulated both through transcription of mRNAs and the subsequent translation into proteins and then also through posttranslational modification of the proteins. Compartmentalization within the cell is critical-location matters. Also, fractions of proteins can be examined-based on secretion, membrane location, phosphorylation, antigenic properties, and other categories.

For decades, proteins have been displayed as "spots" on twodimensional gels, separating proteins by charge and by size. Actually, this approach was very discouraging because so few spots were identified. The genome sequences and the mass spectrometry of proteins have dramatically altered this situation. New methods are appearing rapidly, as noted in other papers of this symposium, to characterize the proteome.

Figure 4 shows results for small-cell carcinoma of the lung, with arrows pointing to protein spots that are significantly upregulated or down-regulated $(2 \times)$ compared to the normal lung master set. ${ }^{10}$ Measuring both protein and gene expression is important, since evidence is accumulating that the two levels are often not closely correlated-because many other factors besides transcription of the gene are important. ${ }^{11}$ These factors include translation, splicing, posttranslational modifications, binding, catabolism, and clearance.

Organizationally, there is now an international Human Proteome Organization (HUPO), patterned after the quite successful Human Genome Organization (HUGO). The founding president of HUPO is Professor Samir Hanash of the University of Michigan. ${ }^{12}$

\section{Breast cancers}

Here I wish to focus on the potential for more specific examination of gene expression and protein expression in al- 

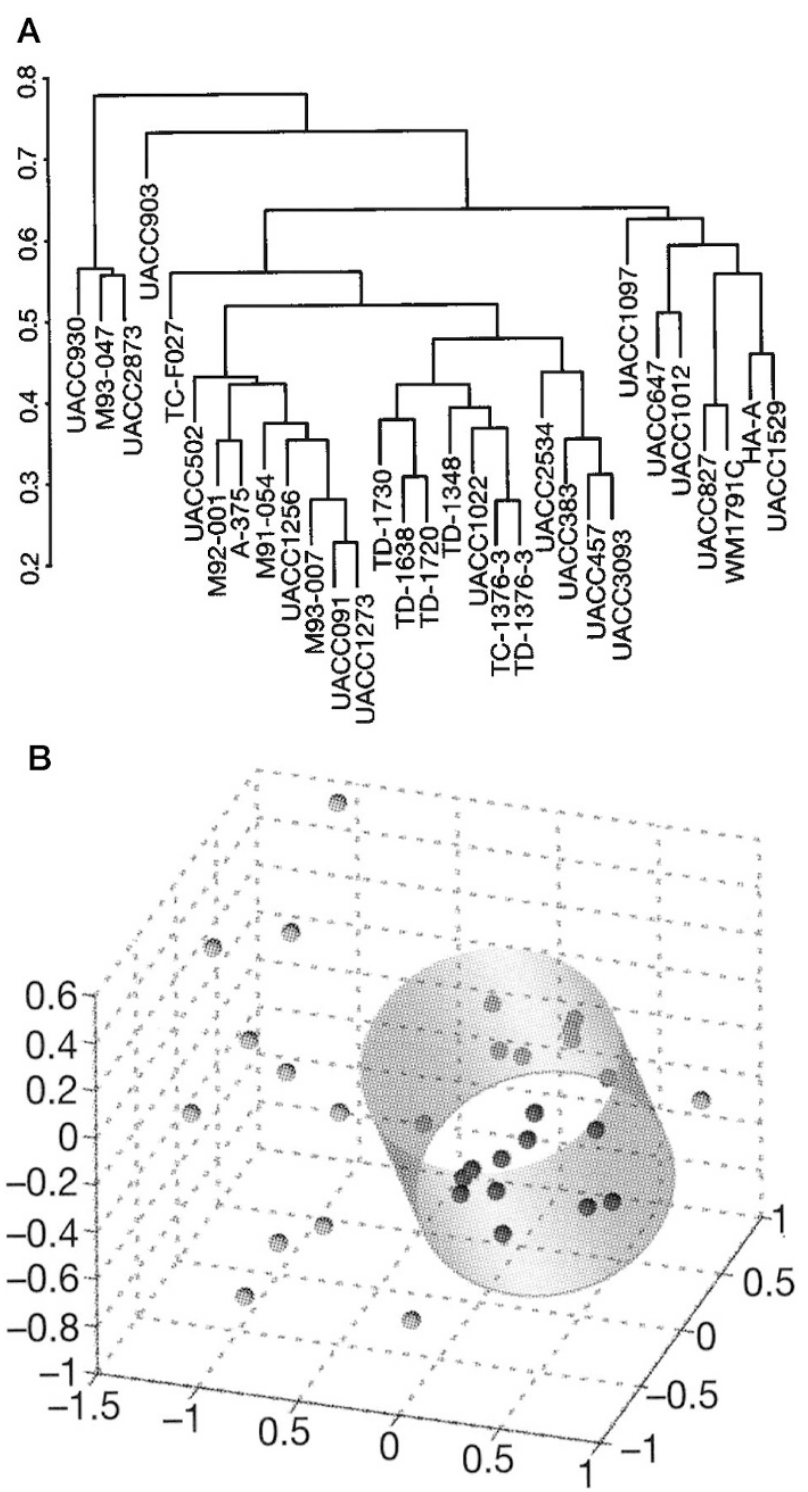

Fig. 3 A: Hierarchical clustering dendrogram of gene expression data for 31 patients with cutaneous malignant melanoma, showing a major cluster of 19 melanomas within a cylindrical region. B: Three-dimensional plot of the same data, showing the major cluster of 19 melanomas within a cylindrical region. Reproduced with permission from Nature, copyright $2000^{9}$ (http://www.nature.com).

ready-recognized forms of breast cancer, ranging from estrogen-receptor-positive versus estrogen-receptor-negative, to HER2/neu-positive versus HER2/neu-negative, to familial $B R C A 1$ versus familial $B R C A 2$ versus sporadic breast cancers. Also, it will be useful to evaluate the influence of tamoxifen and raloxifene on such tumors, possibly by sampling ductal fluid for protein and cells, hopefully clarifying some very preliminary epidemiological findings. ${ }^{13}$ Similar approaches will be helpful for many other treatments.

The most instructive practical result to date is the development of a new drug called Herceptin ${ }^{\circledR}$ (trastuzumab) for advanced breast cancer. The drug represents a "pharmacogenomic prescription." Herceptin was designed to attack the HER2/neu receptor on the surface of breast cancer cells and

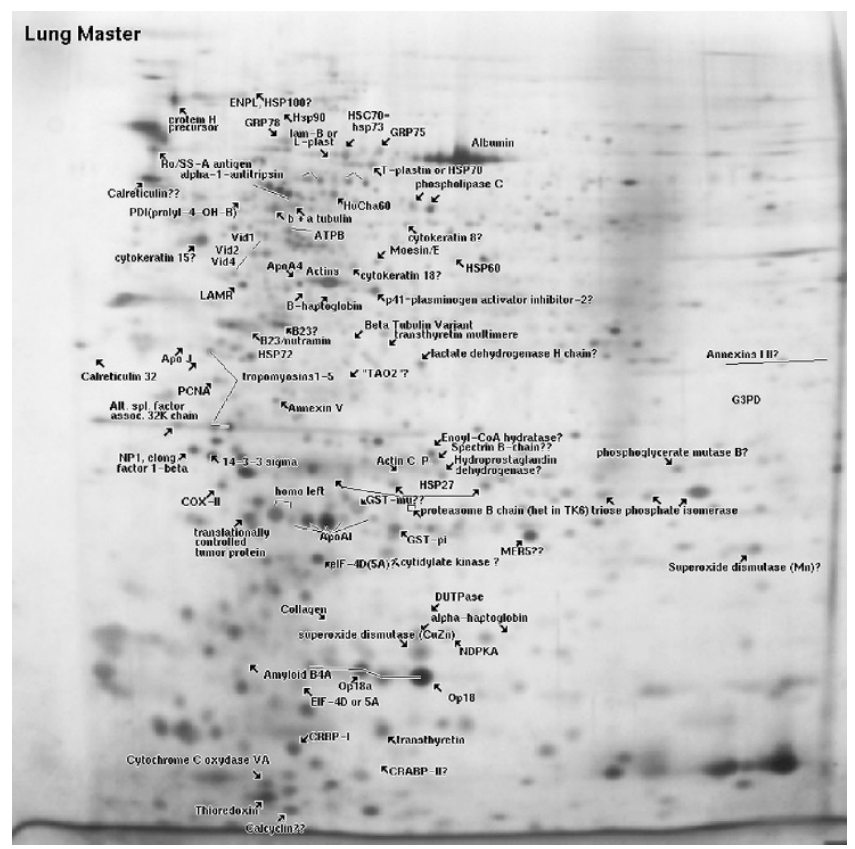

Fig. 4 Lung cancer proteome two-dimensional gel. Based on information from $\mathrm{Oh}$ et al. ${ }^{10}$

works only if that receptor is up-regulated, which occurs in about $30 \%$ of breast cancer patients. ${ }^{14}$ These patients benefit from the expensive new drug; the other $70 \%$ of women with breast cancer who are HER2-negative should not receive this drug. Fluorescence in situ hybridization appears to be the most accurate, reproducible, reliable test for HER2 overexpression in routine diagnostic laboratories. ${ }^{15}$

\section{BIOLOGICAL NANOTECHNOLOGY}

There are other life sciences advances of importance to oncology besides genomics, proteomics, and bioinformatics. One of particular interest is nanotechnology, which has emerged from material sciences and clever organic chemistry. These small "stealth" molecules can penetrate the blood-brain barrier and can evade immune surveillance mechanisms. Perhaps we will need to worry about their toxicological properties, but so far there seem to be useful applications. Molecules built up from relatively simple nitrogen atom units into dendrimers have step functions in size and other properties. ${ }^{15}$ Smart nanodevices can target a tumor, carry imaging capability to document their presence in the tumor, sense pathophysiological defects in tumor cells, deliver therapeutic agents based on the tumor's characteristics, respond to noninvasive external triggers to release the therapeutic agent, document the tumor response, and identify residual tumor cells. ${ }^{16}$ At the Center for Biological Nanotechnology at the University of Michigan, Dr. James Baker and colleagues have a GMP-certified facility for synthesis of these dendrimer nanodevices, cellular assays to demonstrate anticancer activities, experimental animals with 
tumors to test the nanodevices in vivo, and scale-up production capabilities for human phase I clinical trials.

\section{THE ETHICAL CONTEXT}

Based on advances in genomics, proteomics, bioinformatics, and nanotechnology, practical applications in medicine and public health will be many. Besides excellent science and careful trials, we must ensure extensive communication with patients, families, and the broader public; demonstrate compassionate values; appreciate diverse cultures and preferences; be open to new knowledge and alternative views; welcome probing questions; and respond to logic and the public's desire to have greater attention to disease prevention and health promotion, not just earlier diagnosis and more effective treatment of life-threatening diseases. Clearly, the examples from oncology will be reflected in many other clinical specialties, as forecast by Collins and McKusick. ${ }^{17}$

\section{References}

1. International Agency for Research on Cancer. GLOBOSCAN 2000: Cancer incidence, mortality, and prevalence worldwide, IARC Cancer Base No. 5. Lyon, France: IARC Press, 2001.

2. National Cancer Institute Cancer Genome Anatomy Project, 2001 http://cgap.nci.nih.gov.

3. Durbin R, Eddy S, Krogh A, Mitchison G, editors. Biological sequence analysis: probabilistic models of proteins and nucleic acids. Cambridge, England: Cambridge University Press, 1999.

4. Zhao LP, Prentice R, Breeden L. Statistical modeling of large microarray data sets to identify stimulus-response profiles. Proc Natl Acad Sci U S A 2001;98:5631-5636.

5. Thomas JG, Olson JM, Tapscott SJ, Zhao LP. An efficient and robust statistical modeling approach to discover differently expressed genes using genomic expression profiles. Genome Res 2001;11:1227-1236.

6. Hanahan D, Weinberg RA. The hallmarks of cancer. Cell 2000;100:57-70.
7. Alizadeh AA, Eisen MB, Davis RE, Ma C, Lossos IS, Rosenwald A, Boldrick JC, Sabet H, Tran T, Yu X, Powell JI, Yang L, Marti GE, Moore T, Hudson Jr J, Lu L, Lewis DB, Tibshirani R, Sherlock G, Chan WC, Greiner TC, Weisenburger DD, Armitage JO, Warnke R, Levy R, Wilson W, Grever MR, Byrd JC, Botstein D, Brown PO, Staudt LM. Distinct types of diffuse large B-cell lymphoma identified by gene expression profiling. Nature 2000;403:503-511.

8. Dhanasekaran SM, Barrette TR, Ghosh D, Shah R, Varambally S, Kurachi K, Pienta KJ, Rubin MA, Chinnaiyan AM. Delineation of prognostic biomarkers in prostate cancer. Nature 2001;412:822-826.

9. Bittner M, Meltzer P, Chen Y, Jiang Y, Seftor E, Hendrix M, Radmacher M, Simon R, Yakhini Z, Ben-Dor A, Sampas N, Dougherty E, Wang E, Marincola F, Gooden C, Lueders J, Glatfelter A, Pollock P, Carpten J, Gillanders E, Leja D, Dietrich K, Beaudry C, Berens M, Alberts D, Sondak V, Hayward N, Trent J. Molecular classification of cutaneous malignant melanoma by gene expression profiling. Nature 2000;406:536-540.

10. Oh JMC, Brichory F, Puravs E, Kuick R, Wood C, Rouillard JM, Tra J, Kardia S, Beer D, Hanash S. A database of protein expression in lung cancer. Proteomics 2001; 1303-1319.

11. Brichory F, Beer D, LeNaour F, Giordano T, Hanash S. Proteomics-based identification of protein gene product 9.5 as a tumor antigen that induces a humoral immune response in lung cancer. Cancer Res 2001;61:7908-7912.

12. Abbott A. Workshop prepares ground for human proteome project. Nature 2001; 413:763.

13. King M-C, Wieand S, Hale K, Lee M, Walsh T, Owens K, Tait J, Ford L, Dunn BK, Costantino J, Wickerham L, Wolmark N, Fisher B. Tamoxifen and breast cancer incidence among women with inherited mutations in BRCA1 and. BRCA2. JAMA 2001;286:2251-2256.

14. Vogel CL, Cobleigh MA, Tripathy D, Gutheil JC, Harris LN, Fehrenbacher L, Slamon DJ, Murphy M, Novotny WF, Burchmore M, Shak S, Stewart SJ. First-line herceptin ${ }^{\circledR}$ monotherapy in metastatic breast cancer. Oncology 2001;61 (suppl S2): 37-42.

15. Bartlett JM, Going JJ, Mallon EA, Watters AD, Reeves JR, Stanton P, Richmond J, Donald B, Ferrier R, Cooke TG. Evaluating HER2 amplification and overexpression in breast cancer. J Pathol 2001;195:422-428.

16. Baker JR Jr, Quintana A, Piehler L, Banazak-Holl M, Tomalia D, Raczka E. The synthesis and testing of anti-cancer therapeutic nanodevices. Biomed Microdevices 2001;3:61-69.

17. Collins FS, McKusick VA. Implications of the Human Genome Project for medical science. JAMA 2001;285:540-544. 